\title{
Low vitamin B12 and elevated folate status in older adults-a risk factor for cognitive impairment?
}

\author{
E. Laird ${ }^{1}$, H. McNulty ${ }^{2}$, M. Ward ${ }^{2}$, L. Hoey ${ }^{2}$ J.J. Strain ${ }^{2}$, M. Casey ${ }^{3}$, C. Cunningham ${ }^{3}$ and \\ A.M. Molloy \\ ${ }^{1}$ School of Medicine, Trinity College, Dublin 2 Ireland, ${ }^{2}$ Northern Ireland Centre for Food and Health, University of \\ Ulster, Coleraine, BT52 1SA, UK and ${ }^{3}$ The Mercers Institute for Research on Ageing, St James's Hospital, Dublin, \\ Ireland
}

Studies post mandatory folic acid fortification in the US suggest that older adults with high folate but low vitamin B12 status have higher methylmalonic acid (MMA) and homocysteine (tHcy) concentrations as well as a higher risk of cognitive impairment compared to those with low B12 and normal folate status ${ }^{(1-4)}$. However, studies from countries with voluntary but not mandatory folic acid fortification do not support these findings ${ }^{(5,6)}$. Therefore, the aim of this study was to investigate folate and B12 status in relation to cognitive function in a large well-characterised older adult population exposed to a voluntary but liberal food fortification policy.

Participants ( $n$ 5186) were from the Trinity Ulster Department of Agriculture (TUDA) Study, a large study of older Irish adults designed to investigate gene-nutrient interactions in the development of chronic diseases of ageing. Participants were recruited from General Practitioner practices in the Western and Northern Health and Social Care Trusts, Northern Ireland and those attending the memory and bone clinics in the Geriatric Unit of St. James Hospital, Dublin, Ireland. Blood samples were analysed for total serum cobalamin, holotranscobalamin, homocysteine (tHcy), red cell folate (RCF) folate and serum folate. Methylmalonic acid (MMA) determination was performed on a subset of samples ( $n$ 1477). Haematological and renal function data were accessed from the main study database. Cognitive function was assessed by repeatable battery for the assessment of neuropsychological status (RBANS) with a score $\leqslant 80$ indicating cognitive impairment. The population was divided into 4 categories based on serum B12 $(<148$ compared to $\geqslant 148 \mathrm{pmol} / \mathrm{L})$ and serum folate $(<60$ and $\geqslant 60 \mathrm{nmol} / \mathrm{L})$ concentrations.

Participants with either low B12 and low folate or low B12 and high folate status had significantly higher MMA and tHcy concentrations compared to the other groups $(P<0.05)$. Those with low B12 and high folate status $(n$ 54) were significantly older, had a higher frequency of anaemia and cognitive impairment and had a higher use of folic acid supplements than all other groups $(P<0 \cdot 05)$. These observational findings suggest that the classification criterion of low B12/high folate artificially selects the oldest old who are the frailest, most cognitively impaired and the most heavily supplemented with folic acid.

Acknowledgement of Funding: The Irish Department of Agriculture, Food and the Marine (DAFF) and the Northern Ireland Department for Employment \& Learning (DEL) through its "Strengthening the All-Island Research Base" initiative. Funding of MMA analysis: Axis Shield Diagnostics, Dundee, Scotland.

1. Miller JW, Garrod MG, Allen LH et al. (2009) Am J Clin Nutr 90, 1586-1592.

2. Selhub J, Morris MS \& Jacques PF. (2007) Proc Natl Acad Sci USA 104, 19995-20000.

3. Morris MS, Jacques PF, Rosenberg IH et al. (2007) Am J Clin Nutr 85, 193-200.

4. Reynolds E. (2006) Lancet Neurol 5, 949-960.

5. Clarke R, Sherliker P, Hin H et al. (2008) BJN 100, 1054-1059.

6. Mills JL, Carter TC, Scott JM et al. (2011) Am J Clin Nutr 94, 495-500. 